Arkivoc

Free to Authors and Readers
A Platinum Open Access Journal for Organic Chemistry

Paper

DOAJ Seal

Arkivoc 2021, part viii, 277-285

\title{
Synthesis and trypanocidal activity of substituted 2,4-diarylquinoline derivatives
}

\author{
Kola A. Oluwafemi ${ }^{a}+$, Siyolise Phunguphungua , Sinalo Gqunu ${ }^{a}$, Michelle Isaacs, ${ }^{b}$ Heinrich C. Hoppe ${ }^{b, c}$, \\ Rosalyn Klein, a,b* and Perry T. Kaye ${ }^{a, b^{*}}$
}

${ }^{a}$ Department of Chemistry, Rhodes University, Makhanda/Grahamstown, 6140, South Africa

${ }^{b}$ Centre for Chemico- and Biomedical Research, Rhodes University, Makhanda/Grahamstown, 6140, South

Africa

'Department of Biochemistry and Microbiology, Rhodes University, Makhanda/Grahamstown, 6140, South

Africa

Email: r.klein@ru.ac.za; p.kaye@ru.ac.za

Received 02-24-2021

Accepted Manuscript 05-17-2021

Published on line 06-27-2021

\section{Abstract}

A small library of nine, novel 2,4-diarylquinoline derivatives has been prepared in high yield via convenient one- or two-step routes from a series of substituted 2-aminobenzophenones. None of the products exhibited toxicity at $20 \mu \mathrm{M}$ against human cervix adenocarcinoma (HeLa) cells, while many of them exhibited encouraging trypanocidal activity against $T$. brucei brucei (a parasite responsible for African cattle trypanosomiasis) - some with $\mathrm{IC}_{50}$ values in the range $2.8-6.2 \mu \mathrm{M}$.
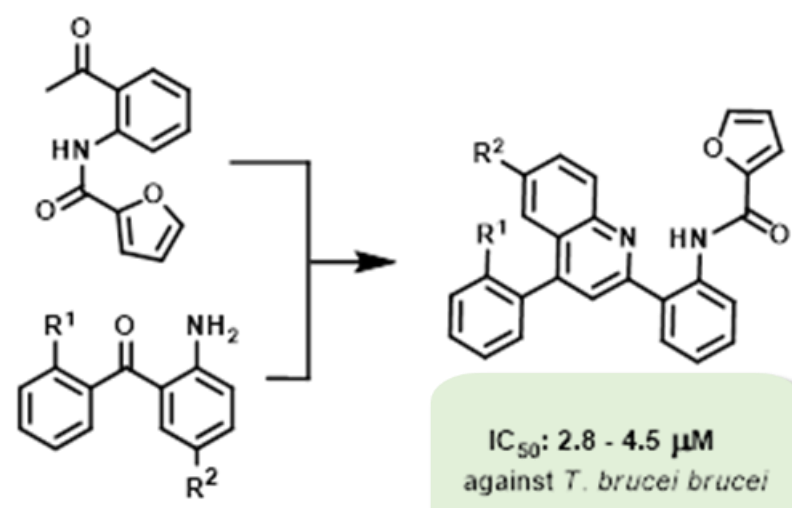

Keywords: Synthesis, trypanocidal activity, diarylquinolines, T. brucei brucei 


\section{Introduction}

African trypanosomiasis (Sleeping Sickness), regarded as one of the neglected tropical diseases (NTDs), is caused by parasitic infection by Trypanosoma species and is transmitted by Tsetse flies (Glossina spp.) which are endemic in Africa. Human African Trypanosomiasis (HAT) and Animal African Trypanosomiasis (AAT) are debilitating diseases which may prove fatal if not treated. ${ }^{1-3}$ Efforts to eradicate the disease have included the use of nifurtimox 1, pentamidine 2 and suramin 3. ${ }^{4-6}$ Trypanothione reductase, farnesyl diphosphate synthase, 6-phospho-gluconate dehydrogenase, and UDP 4'-galactose epimerase are examples of trypanosomal enzymes currently receiving attention as drug targets. ${ }^{7}$ However, the range of available trypanocidal drugs is very limited; many have been in use for a long time and the development of resistance has been reported. ${ }^{4-}$ 6;8;9 There is, therefore, an urgent need to discover new and effective alternatives. ${ }^{4}$ Plasmodium falciparum, which is responsible for malaria, is also a parasitic protozoan, and various quinoline derivatives are well known for their antimalarial activity. ${ }^{10-16}$ In this communication, we report the synthesis of a series of novel 2,4diarylquinoline derivatives and their evaluation for trypanocidal activity.

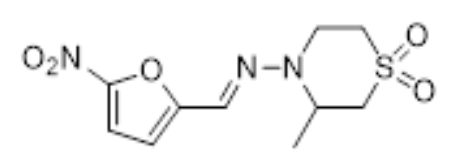

1

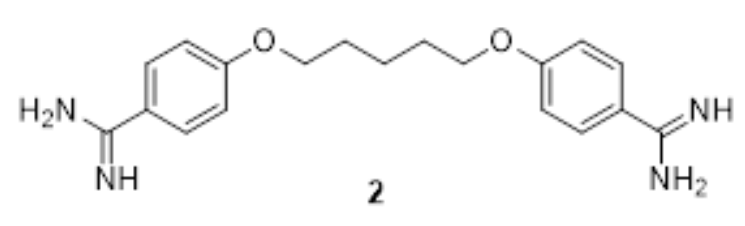

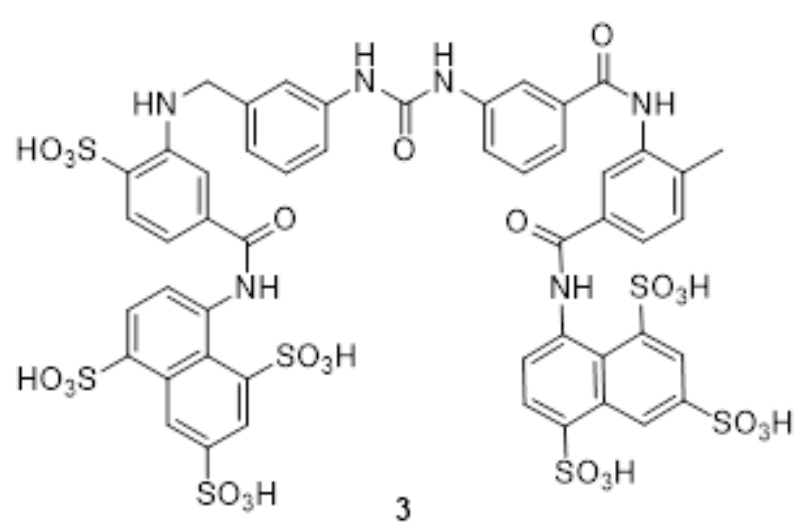

Figure 1. Structures of selected drugs used for the treatment of trypanosomiasis.

\section{Results and Discussion}

Access to both sets of 2,4-diarylquinoline derivatives $\mathbf{4 a - g}$ and $5 \mathrm{~h}, \mathrm{i}$ was achieved by adopting Friedländer-type methodology, involving the reaction of the common, 2-aminobenzophenones (6) with the variously substituted acetophenones (7) and (8), respectively (Scheme 1). Thus, in an approach similar to that used by Sankaran et al. $^{17}$ in their synthesis of biquinoline- and quinoline-bearing chromenes, equal equivalents of the appropriate acetophenones (7) and 2-aminobenzophenones (6) were reacted in refluxing acetic acid in the presence of a catalytic quantity of sulphuric acid to give the desired substituted 2,4-diarylquinolines 4a-g in yields ranging from 80 to $95 \%$ (Table 1 ).

The acetophenone component, $\mathrm{N}$-(2-acetylphenyl)furan-2-carboxamide $\mathbf{8}$, required for the synthesis of the 2-[2-(furoylamino)phenyl]quinoline analogues $5 \mathrm{~h}, \mathrm{i}$ was specially prepared by reacting 0 aminoacetophenone $\mathbf{9}$ with furoyl chloride $\mathbf{1 0}$ using potassium carbonate as a base in dichloromethane at room temperature. Subsequent reaction of compound 8 with the appropriate 2-aminobenzophenones (6) afforded the corresponding the 2-[2-(furoylamino)phenyl]quinolines $5 \mathbf{i}, \mathbf{j}$ in excellent yields (90-92\%; Table 1 ). 
Toxicity studies conducted against HeLa (human cervix adenocarcinoma) cells using a resazurin-based fluorescence assay, indicated that none of the synthesised compounds $\mathbf{4 a - g}$ and $\mathbf{5 h}$, i showed any cytotoxicity at a concentration of $20 \mu \mathrm{M}$ (Table 1 and Figure 2). On testing against T. brucei brucei, however, most of the compounds exhibited a measure of anti-trypanosomal activity - a number of them with very encouraging $\mathrm{IC}_{50}$ values $(2.8-6.2 \mu \mathrm{M}$; Table 1 and Figure 3).

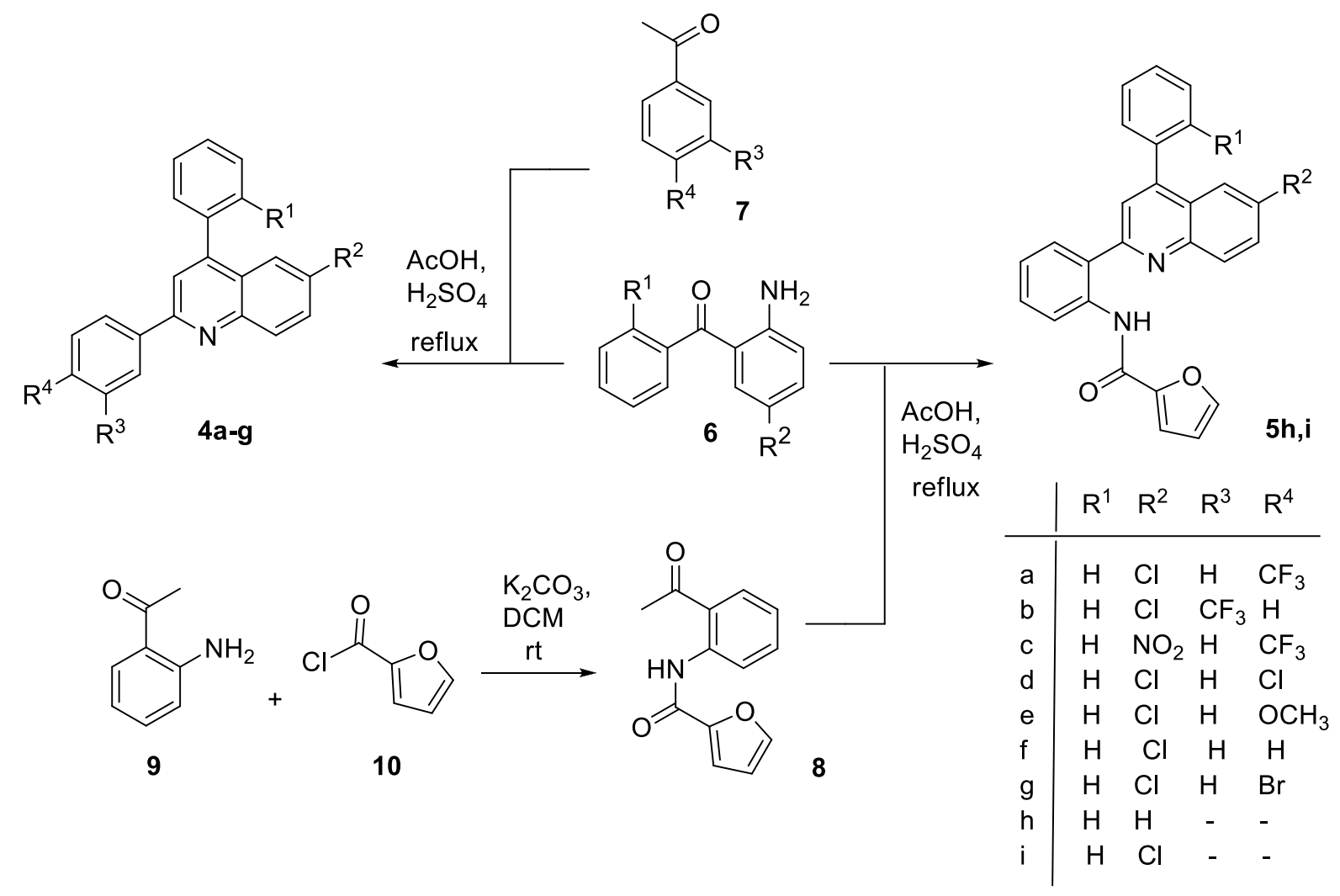

Scheme 1. Synthesis of 2,4-diarylquinolines 4 a-g and $5 \mathrm{~h}, \mathrm{i}$.

The bioassay data reveal a number of significant structure-activity relationship patterns.

i) The 2-[2-(furoylamino)phenyl]quinoline derivatives $\mathbf{5 h}$ and $\mathbf{5 i}$ both exhibited encouraging activity against $T$. brucei brucei at very low micromolar concentrations (IC 50 : $2.8-4.5 \mu \mathrm{M})$, whereas only one of the 2-phenylquinoline analogues showed comparable activity (4b: $I C_{50}: 6.2 \mu \mathrm{M}$ ).

ii) Compound $\mathbf{4 b}$ is the only 2 -phenylquinoline derivative to contain a meta-trifluoromethyl group $\left(R^{3}\right)$ on the D-ring; moving the trifluoromethyl group $\left(R^{3}\right)$ to the para-position, as in compound $4 \mathbf{a}$, decreases activity, increasing $\%$ T. brucei brucei viability from $6 \%$ for $\mathbf{4 b}$ to $52 \%$ for $4 \mathbf{a}$.

iii) Changing the B-ring substituent $\left(R^{2}\right)$ from $\mathrm{Cl}$ in 4 a to the strongly electron-withdrawing nitro group in compound 4c effectively nullifies anti-trypanosomal activity with \% $T$. brucei brucei viability increasing from $52 \%$ for $4 a$ to essentially $100 \%$ for $4 c$.

iv) Compounds $\mathbf{4 a}, \mathbf{4 b}, \mathbf{4 d}$ and $\mathbf{4 e}$, which contain the same B-ring substituent $\left(\mathrm{R}^{2}=\mathrm{Cl}\right)$ but differ in the nature of their D-ring substituents $\left(\mathrm{R}^{4}=\mathrm{CF}_{3}, \mathrm{Cl}\right.$ and $\mathrm{OCH}_{3}$, respectively) all show activity against $T$. brucei brucei, but when $\mathrm{R}^{4}$ is changed to $\mathrm{Br}$, as in compound $\mathbf{4 g}$, activity is lost altogether. 
Table 1. Effect of compounds $\mathbf{4 a - g}$ and $\mathbf{5 h}, \mathbf{i}$ at $20 \mu \mathrm{M}$ on the viability of HeLa and T.brucei brucei cells

\begin{tabular}{|c|c|c|c|c|c|c|c|}
\hline & $\mathbf{R}^{1}$ & $\mathbf{R}^{2}$ & $\mathbf{R}^{3}$ & $\mathbf{R}^{4}$ & $\begin{array}{c}\% \\
\text { Yield }\end{array}$ & $\begin{array}{c}\%^{b, c} \\
\text { HeLa cell } \\
\text { viability }\end{array}$ & $\begin{array}{c}\%^{c, d} \\
\text { T. brucei } \\
\text { Viability }\end{array}$ \\
\hline $4 a$ & $\mathrm{H}$ & $\mathrm{Cl}$ & $\mathrm{H}$ & $\mathrm{CF}_{3}$ & 90 & $110(1)$ & $52(3)$ \\
\hline $4 b$ & $\mathrm{H}$ & $\mathrm{Cl}$ & $\mathrm{CF}_{3}$ & $\mathrm{H}$ & 87 & 100 (1) & $\begin{array}{l}6(2) \\
(6.2 \mu \mathrm{M})^{\mathrm{a}}\end{array}$ \\
\hline $4 c$ & $\mathrm{H}$ & $\mathrm{NO}_{2}$ & $\mathrm{H}$ & $\mathrm{CF}_{3}$ & 80 & $113(6)$ & 107 (4) \\
\hline $4 d$ & $\mathrm{H}$ & $\mathrm{Cl}$ & $\mathrm{H}$ & $\mathrm{Cl}$ & 93 & $102(1)$ & $66(4)$ \\
\hline $4 e$ & $\mathrm{H}$ & $\mathrm{Cl}$ & $\mathrm{H}$ & $\mathrm{OCH}_{3}$ & 94 & $113(5)$ & $43(5)$ \\
\hline $4 f$ & $\mathrm{H}$ & $\mathrm{Cl}$ & $\mathrm{H}$ & $\mathrm{H}$ & 93 & $116(7)$ & $73(8)$ \\
\hline $4 g$ & $\mathrm{H}$ & $\mathrm{Cl}$ & $\mathrm{H}$ & $\mathrm{Br}$ & 95 & 107 (9) & $104(0.01)$ \\
\hline $5 h$ & $\mathrm{H}$ & $\mathrm{H}$ & - & - & 92 & $117(4)$ & $\begin{array}{l}0.5(1) \\
(4.5 \mu \mathrm{M})^{\mathrm{a}}\end{array}$ \\
\hline $5 i$ & $\mathrm{H}$ & $\mathrm{Cl}$ & - & - & 90 & $117(6)$ & $\begin{array}{l}0.5(0.5) \\
(2.8 \mu \mathrm{M})^{\mathrm{a}}\end{array}$ \\
\hline
\end{tabular}

${ }^{\mathrm{a}} \mathrm{IC} \mathrm{C}_{50}$ values in parentheses. ${ }^{\mathrm{b}}$ Emetine control: $\mathrm{IC} \mathrm{C}_{50}=0.05 \mu \mathrm{M}$. ${ }^{\mathrm{c}}$ Standard deviation in parentheses.

d Pentamidine control: $\mathrm{IC}_{50}=0.005 \mu \mathrm{M}$.

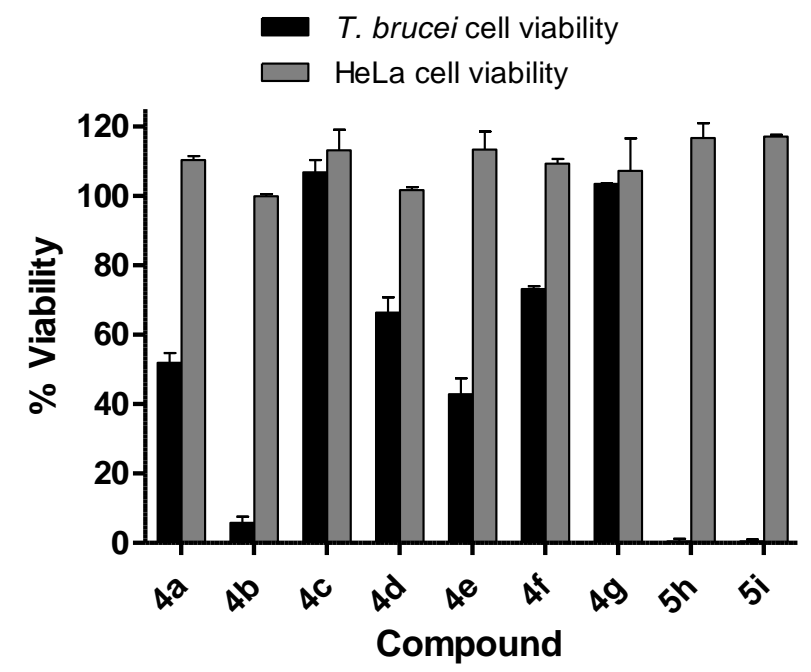

Figure 2. Graphical display of \% HeLa and T.brucei brucei cell viability data with compounds 4 a-g and $5 \mathbf{h}, \mathbf{i}$ at 20 $\mu \mathrm{M}$. 


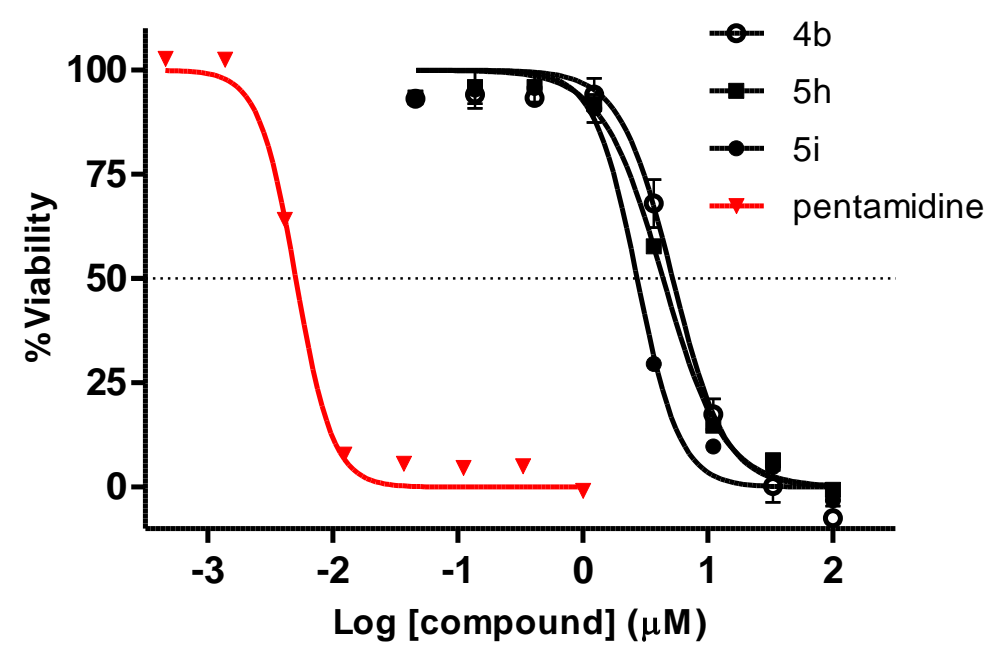

Figure 3. Dose-response curves showing the \% viability of T.brucei brucei cells at various concentrations of compounds $\mathbf{4 b}, \mathbf{5} \mathbf{h}$ and $\mathbf{5 i}$ and the control, pentamidine.

\section{Conclusions}

In conclusion, convenient synthetic access to library of substituted 2,4-diphenylquinolines has been established and, although the mode of action is not known at present, it is apparent that a number of these compounds exhibit very encouraging anti-trypanosomal activity. Interesting structure-activity relationships have been identified, and the 2-[2-(furoylamino)phenyl]quinoline derivatives $\mathbf{5 h}, \mathbf{i}$, in particular, are promising lead compounds for the development of novel trypanocidal agents.

\section{Experimental Section}

General. All chemicals were used as purchased from Sigma-Aldrich Chemical Co. Analytical thin layer chromatography (TLC) was performed using pre-coated silica gel plates. NMR spectra were recorded on Bruker 300, 400 and $600 \mathrm{MHz}$ NMR spectrometers, chemical shifts were calibrated relative to the residual proton signal in DMSO- $d_{6}(2.5 \mathrm{ppm})$ and the NMR spectra were analysed using Mestrenova. IR Spectra were recorded on a Perkin Elmer Spectrum 100 FTIR spectrometer with a diamond window. Melting points were determined using a hot-stage apparatus and are uncorrected. HRMS analyses were conducted at Rhodes University and by the Central Analytical Facilities Unit at the University of Stellenbosch.

Representative synthetic methods, full characterisation data for the other novel compounds, and Bioassay protocols are detailed below. Bioassay reports and NMR spectra are provided in the Supplementary Material file.

$\mathbf{N}$-(2-Acetylphenyl)furan-2-carboxamide 8. Potassium carbonate (172 $\mathrm{mg}, 1.5 \mathrm{eq}$.) was added to a solution of $o$-aminoacetophenone $(1 \mathrm{~mL}, 8.3 \mathrm{mmol})$ in dichloromethane $(30 \mathrm{~mL})$, and the mixture was stirred at room temperature for 30 minutes. Furoyl chloride $(0.8 \mathrm{~mL}, 8.3 \mathrm{mmol})$ was then added and the resulting mixture 
stirred at room temperature for 1 hour, with the reaction progress being monitored by TLC. After the completion of the reaction, the mixture was extracted into DCM $(2 \times 60 \mathrm{~mL})$, the organic solutions were combined, washed with deionized water $(4 \times 60 \mathrm{~mL})$, dried with anhydr. potassium sulphate and filtered. The solvent was removed in vacuo and the residue was purified using column chromatography on silica gel [elution with ethyl acetate-hexane (2:1] to afford $\mathbf{N}$-(2-acetylphenyl)furan-2-carboxamide (8). White solid (1.6 g, 84\%), $\mathrm{mp} 52-54{ }^{\circ} \mathrm{C}$ (Found: $\mathrm{MH}^{+}, \mathrm{m} / z$ 230.0854. $\mathrm{C}_{13} \mathrm{H}_{12} \mathrm{NO}_{3}$ requires 230.0817); $\delta_{\mathrm{H}} / \mathrm{ppm}\left(600 \mathrm{MHz}\right.$; DMSO- $d_{6}$ ) 12.45 $(1 \mathrm{H}, \mathrm{s}, \mathrm{NH}), 8.63(1 \mathrm{H}, \mathrm{d}, J 8.4 \mathrm{~Hz}, \mathrm{ArH}), 8.10(1 \mathrm{H}, \mathrm{dd}, J$ 7.9, $0.9 \mathrm{~Hz}, \mathrm{ArH}), 7.99(1 \mathrm{H}, \mathrm{s}, \mathrm{ArH}), 7.64(1 \mathrm{H}, \mathrm{m}, \mathrm{ArH})$, $7.28(1 \mathrm{H}, \mathrm{d}, J 3.4 \mathrm{~Hz}, \mathrm{ArH}), 7.24(1 \mathrm{H}, \mathrm{m}, \mathrm{ArH}), 6.74(1 \mathrm{H}, \mathrm{dd}, J 3.4,1.7 \mathrm{~Hz}, \mathrm{ArH})$ and $2.68\left(3 \mathrm{H}, \mathrm{s}, \mathrm{COCH}_{3}\right) ; \delta_{c} / \mathrm{ppm}$ $\left(150 \mathrm{MHz}\right.$; DMSO- $\left.d_{6}\right) 203.5$ and 156.2 (C=O), 147.5, 146.4, 139.4, 134.9, 132.5, 123.2, 122.8, 120.1, 115.8 and $112.9(\mathrm{ArC})$ and $28.8\left(\mathrm{CH}_{3}\right)$.

The general procedure for the synthesis of the 2,4 -diarylquinolines $(4 a-g)$ and $(5 \mathrm{~h}, \mathrm{i})$ is illustrated by the following example. Conc. sulphuric acid (0.2 mL; CAUTION!) was added to a solution of 2-amino-5chlorobenzophenone $(150.6 \mathrm{mg}, 0.7 \mathrm{mmol})$ and 4'-(trifluoromethyl)acetophenone $(122.3 \mathrm{mg}, 0.7 \mathrm{mmol}) \mathrm{in}$ acetic acid $(10 \mathrm{~mL})$. The resulting solution was stirred under a reflux condenser at $80{ }^{\circ} \mathrm{C}$ for 2 hours, during which the reaction progress was monitored by TLC. After completion of the reaction, the crude mixture was cooled to room temperature and then poured into ice-cold deionized water $(150 \mathrm{~mL})$. The resulting precipitate was filtered off, washed with cold deionized water $(2 \times 100 \mathrm{~mL})$ and dried at room temperature for 24 hours. The dried material was washed with ethyl acetate $(3 \times 30 \mathrm{~mL})$ to afford essentially pure 6-chloro-2-[4(trifluoromethyl)phenyl]-4-phenylquinoline (4a). Yellow solid (242 mg, 90\%), m.p.122-126 ${ }^{\circ} \mathrm{C}$ (Found: $\mathrm{MH}^{+}$, $\mathrm{m} / \mathrm{z}$ 384.0770. $\mathrm{C}_{22} \mathrm{H}_{14} \mathrm{ClF}_{3} \mathrm{~N}$ requires 384.0767); $\delta_{\mathrm{H}} / \mathrm{ppm}\left(600 \mathrm{MHz}\right.$; DMSO- $\left.d_{6}\right) 8.56(2 \mathrm{H}, \mathrm{d}, J 8.3 \mathrm{~Hz}, \mathrm{ArH}), 8.23$ $(1 \mathrm{H}, \mathrm{d}, J 9.0 \mathrm{~Hz}, \mathrm{ArH}), 8.20(1 \mathrm{H}, \mathrm{s}, \mathrm{ArH}), 7.91(2 \mathrm{H}, \mathrm{d}, J 8.3 \mathrm{~Hz}, \mathrm{ArH}), 7.87(1 \mathrm{H}, \mathrm{dd}, J$ 9.0, $2.3 \mathrm{~Hz}, \operatorname{ArH}), 7.80(1 \mathrm{H}, \mathrm{d}, J$ $2.3 \mathrm{~Hz}, \mathrm{ArH}), 7.68$ - 7.59 (5H, overlapping m, ArH); $\delta \mathrm{c} / \mathrm{ppm}$ (150 MHz; DMSO-d $d_{6}$ 155.2, 148.9, 147.0, 142.4, 137.1, 132.6, 132.4, 131.2, 130.1 (d, $J_{C, F}=31.7 \mathrm{~Hz}$ ), 130.0, 129.5, 129.4, 128.9 (d, $\left.J_{C, F}=12.5 \mathrm{~Hz}\right), 128.7,126.7$, $126.2\left(\mathrm{dd}, J_{\mathrm{C}, \mathrm{F}}=7.6,3.8 \mathrm{~Hz}\right), 124.4$ and $120.5[\operatorname{ArC}]$.

\section{Analytical data for other new compounds $(4 \mathrm{~b}-\mathrm{g})$ and $(5 \mathrm{~h}, \mathrm{i})$}

6-Chloro-2-[3-(trifluoromethyl)phenyl]-4-phenylquinoline (4b). Yellow solid (234 mg, 87\%), $\mathrm{mp} 108-110{ }^{\circ} \mathrm{C}$ (Found: $\mathrm{MH}^{+}, \mathrm{m} / \mathrm{z}$ 384.0773. $\mathrm{C}_{22} \mathrm{H}_{14} \mathrm{ClF}_{3} \mathrm{~N}$ requires 384.0767); $\delta_{\mathrm{H}} / \mathrm{ppm}\left(600 \mathrm{MHz}\right.$; DMSO- $\left.d_{6}\right) 8.69(1 \mathrm{H}, \mathrm{s}, \mathrm{ArH})$, $8.66(1 \mathrm{H}, \mathrm{d}, J 7.9 \mathrm{~Hz}, \mathrm{ArH}), 8.26(1 \mathrm{H}, \mathrm{s}, \mathrm{ArH}), 8.23(1 \mathrm{H}, \mathrm{d}, J 9.0 \mathrm{~Hz}, \mathrm{ArH}), 7.89(1 \mathrm{H}, \mathrm{d}, J 7.9 \mathrm{~Hz}, \operatorname{ArH}), 7.85(1 \mathrm{H}, \mathrm{dd}$, J 9.0, $2.3 \mathrm{~Hz}, \mathrm{ArH})$ and 7.8-7.6 (7H, series of overlapping signals, $\mathrm{ArH}) ; \delta \mathrm{c} / \mathrm{ppm}\left(150 \mathrm{MHz}\right.$; DMSO- $\left.d_{6}\right)$ 154.6, $148.4,146.5,139.1,136.7,132.1,131.8,131.4,130.7,130.1,129.9,129.6,129.0,128.9,126.4$ (d, J $3.5 \mathrm{~Hz}$ ), 126.2, 123.93, 123.85 (d, J $3.8 \mathrm{~Hz}$ ), 123.4 (d, J 3.2 Hz), 119.8 [ArC].

6-Nitro-2-[4-(trifluoromethyl)phenyl]-4-phenylquinoline (4c). Yellow solid (126.2 mg, 80\%), mp $192-194{ }^{\circ} \mathrm{C}$ (Found: $\mathrm{MH}^{+}, \mathrm{m} / \mathrm{z}$ 395.1004. $\mathrm{C}_{22} \mathrm{H}_{14} \mathrm{~F}_{3} \mathrm{~N}_{2} \mathrm{O}_{2}$ requires 395.1007); $\delta_{\mathrm{H}} / \mathrm{ppm}\left(600 \mathrm{MHz}\right.$; DMSO-d $\left.d_{6}\right) 8.71(1 \mathrm{H}, \mathrm{s}, \mathrm{ArH})$, $8.63(2 \mathrm{H}, \mathrm{d}, J 7.0 \mathrm{~Hz}, \mathrm{ArH}), 8.54(1 \mathrm{H}, \mathrm{d}, J 9.1 \mathrm{~Hz}, \mathrm{ArH}), 8.39(1 \mathrm{H}, \mathrm{d}, J 9.1 \mathrm{~Hz}, \mathrm{ArH}), 8.37(1 \mathrm{H}, \mathrm{s}, \operatorname{ArH}), 7.94(2 \mathrm{H}, \mathrm{d}, J$ $7.3 \mathrm{~Hz}, \mathrm{ArH}), 7.75(2 \mathrm{H}, \mathrm{d}, J 6.0 \mathrm{~Hz}, \mathrm{ArH})$ and 7.72-7.63 (3H, m, ArH); $\delta_{c} / p p m(150 \mathrm{MHz}$; DMSO-d $) 151.20(\mathrm{~m})$, 150.6, $150.2(\mathrm{~m}), 145.4\left(\mathrm{~d}, J_{C, F}=6.6 \mathrm{~Hz}\right), 141.5\left(\mathrm{~d}, J_{C, F}=5.6 \mathrm{~Hz}\right), 136.2,131.8,129.8,129.5,129.1,128.7,125.9$ (d, J $3.5 \mathrm{~Hz}), 124.5,123.5,122.5$ and 120.8 [ArC].

6-Chloro-2-(4-chlorophenyl)-4-phenylquinoline (4d). White solid (140.1 mg, $93 \%$ ), mp 160-162 ${ }^{\circ} \mathrm{C}$ (Found: $\mathrm{MH}^{+}, \mathrm{m} / \mathrm{z}$ 350.0506. $\mathrm{C}_{21} \mathrm{H}_{14} \mathrm{Cl}_{2} \mathrm{~N}$ requires 350.0503); $\delta_{\mathrm{H}} / \mathrm{ppm}\left(300 \mathrm{MHz}\right.$; DMSO- $\left.d_{6}\right) 8.39(2 \mathrm{H}, \mathrm{d}, J 8.6 \mathrm{~Hz}, \mathrm{ArH})$, $8.19(1 \mathrm{H}, \mathrm{d}, J 9.0 \mathrm{~Hz}, \mathrm{ArH}), 8.14(1 \mathrm{H}, \mathrm{s}, \mathrm{ArH}), 7.85(1 \mathrm{H}, \mathrm{dd}, J$ 9.0, $2.4 \mathrm{~Hz}, \mathrm{ArH}), 7.78(1 \mathrm{H}, \mathrm{d}, J 2.4 \mathrm{~Hz}, \mathrm{ArH})$ and 7.7$7.6(7 \mathrm{H}, \mathrm{m}, \mathrm{ArH}) ; \delta_{c} / \mathrm{ppm}\left(75 \mathrm{MHz}\right.$; DMSO-d $\left.d_{6}\right) 155.0,148.2,146.5,136.9,136.7,134.9,131.9,131.5,130.5$, $129.6,129.2,129.0,128.9,126.0,123.9$ and $119.6(\operatorname{ArC})$. 
6-Chloro-2-(4-methoxyphenyl)-4-phenylquinoline (4e). White solid (228 mg, $94 \%), \mathrm{mp} 128-130{ }^{\circ} \mathrm{C}$ (Found: $\mathrm{MH}^{+}, \mathrm{m} / z$ 346.1000. $\mathrm{C}_{22} \mathrm{H}_{17} \mathrm{ClNO}$ requires 346.0999); $\delta_{\mathrm{H}} / \mathrm{ppm}\left(400 \mathrm{MHz} ; \mathrm{DMSO}-d_{6}\right) 8.28(2 \mathrm{H}, \mathrm{d}, J 8.8 \mathrm{~Hz}, \mathrm{ArH})$, $8.11(1 \mathrm{H}, \mathrm{d}, J 9.0 \mathrm{~Hz}, \mathrm{ArH}), 8.01(1 \mathrm{H}, \mathrm{s}, \mathrm{ArH}), 7.77(1 \mathrm{H}, \mathrm{dd}, J$ 9.0, $2.3 \mathrm{~Hz}, \mathrm{ArH}), 7.71(1 \mathrm{H}, \mathrm{d}, J 2.2 \mathrm{~Hz}, \mathrm{ArH}), 7.66-$ $7.55(5 \mathrm{H}, \mathrm{m}, \mathrm{ArH}), 7.08(2 \mathrm{H}, \mathrm{d}, J 8.8 \mathrm{~Hz}, \mathrm{ArH})$ and $3.83\left(3 \mathrm{H}, \mathrm{s}, \mathrm{OCH}_{3}\right) ; \delta_{c} / p p m(100 \mathrm{MHz}$; DMSO-d $)$ 161.0, 156.0, $147.9,146.7,137.0,131.8,130.8,130.6,130.4,129.6,129.01,128.99,128.95,125.7,123.9,119.3,114.3$ (ArC) and $55.4\left(\mathrm{OCH}_{3}\right)$.

6-Chloro-2,4-diphenylquinoline (4f). White solid (256.3 mg, 93\%), mp 118-120 ${ }^{\circ} \mathrm{C}$ (Found: $\mathrm{MH}^{+}, \mathrm{m} / z 316.0934$. $\mathrm{C}_{21} \mathrm{H}_{15} \mathrm{ClN}$ requires 316.0893); $\delta_{\mathrm{H}} / \mathrm{ppm}\left(400 \mathrm{MHz}\right.$; DMSO- $\left.d_{6}\right) 8.33(2 \mathrm{H}, \mathrm{d}, J 6.6 \mathrm{~Hz}, \mathrm{ArH}), 8.19(1 \mathrm{H}, \mathrm{d}, J 9.0 \mathrm{~Hz}$, $\operatorname{ArH}), 8.09(1 \mathrm{H}, \mathrm{s}, \mathrm{ArH}), 7.83(1 \mathrm{H}, \mathrm{dd}, J$ 9.0, $2.3 \mathrm{~Hz}, \mathrm{ArH}), 7.77(1 \mathrm{H}, \mathrm{d}, J 2.3 \mathrm{~Hz}, \operatorname{ArH})$ and $7.66-7.52(8 \mathrm{H}$, overlapping $\mathrm{m}, \mathrm{ArH})$.

2-(4-Bromophenyl)-6-chloro-4-phenylquinoline (4g). White solid (150 mg, 95\%); mp 166-168 ${ }^{\circ} \mathrm{C}\left(\right.$ Found: $\mathrm{MH}^{+}$, $m / z$ 393.9993. $\mathrm{C}_{21} \mathrm{H}_{14} \mathrm{BrClN}$ requires 393.9998); $\delta_{\mathrm{H}} / \mathrm{ppm}\left(600 \mathrm{MHz}\right.$; DMSO- $\left.d_{6}\right) 8.30(2 \mathrm{H}, \mathrm{d}, J 8.0 \mathrm{~Hz}, \mathrm{ArH}), 8.19$ $(1 \mathrm{H}, \mathrm{d}, J 9.0 \mathrm{~Hz}, \mathrm{ArH}), 8.11(1 \mathrm{H}, \mathrm{s}, \mathrm{ArH}), 7.84(1 \mathrm{H}, \mathrm{d}, J 9.0 \mathrm{~Hz}, \mathrm{ArH}), 7.77(1 \mathrm{H}, \mathrm{s}, \mathrm{ArH}), 7.75(2 \mathrm{H}, \mathrm{d}, J 8.0 \mathrm{~Hz}, \mathrm{ArH})$

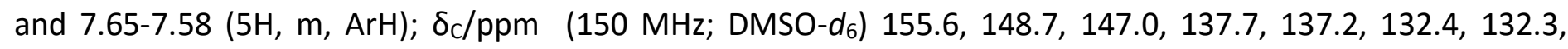
132.0, 131.1, 130.0, 129.9, 129.5, 129.4, 126.5, 124.4, 124.2 and 120.0 (ArC).

2-[2-(Furan-2-carboxamido)phenyl]-4-phenylquinoline (5h). Gray solid (180 mg, 92\%), mp $176-180{ }^{\circ} \mathrm{C}$ (Found: $\mathrm{MH}^{+}, m / z$ 391.1453. $\mathrm{C}_{26} \mathrm{H}_{19} \mathrm{~N}_{2} \mathrm{O}_{2}$ requires 391.1447); $\delta_{\mathrm{H}} / \mathrm{ppm}\left(600 \mathrm{MHz}\right.$; DMSO- $\left.d_{6}\right) 13.82(1 \mathrm{H}, \mathrm{s}, \mathrm{NH}), 8.62(1 \mathrm{H}$, d, J $8.1 \mathrm{~Hz}, \mathrm{ArH}), 8.40(1 \mathrm{H}, \mathrm{d}, J 8.4 \mathrm{~Hz}, \mathrm{ArH}), 8.22(1 \mathrm{H}, \mathrm{d}, J 7.5 \mathrm{~Hz}, \mathrm{ArH}), 8.10(1 \mathrm{H}, \mathrm{s}, \mathrm{ArH}), 8.04(1 \mathrm{H}, \mathrm{s}, \mathrm{ArH}), 7.98$ $(1 \mathrm{H}, \mathrm{m}, \mathrm{ArH}), 7.9-7.3(8 \mathrm{H}$, series of overlapping $\mathrm{m}, \mathrm{ArH}), 7.32(2 \mathrm{H}, \mathrm{m}, \mathrm{ArH})$ and $6.77(1 \mathrm{H}, \mathrm{s}, \mathrm{ArH}) ; \delta \mathrm{c} / \mathrm{ppm}(150$ MHz; DMSO-d $)_{6} 156.8$ (C=O), 155.8, 148.1, 145.8, 137.5, 137.1, 130.85, 130.84, 130.5, 130.3, 129.6, 128.9, $128.8,128.6,127.6,125.4,125.3,124.6,124.0,121.3,120.9,115.3,112.8$ and $99.6(\operatorname{ArC})$.

6-Chloro-2-[2-(furan-2-carboxamido)phenyl]-4-phenylquinoline (5i). Gray solid (191 mg, 90\%), mp $150-156{ }^{\circ} \mathrm{C}$ (Found: $\mathrm{MH}^{+}, m / z$ 425.1053. $\mathrm{C}_{26} \mathrm{H}_{18} \mathrm{ClN}_{2} \mathrm{O}_{2}$ requires 425.1057); $\delta_{\mathrm{H}} / \mathrm{ppm}\left(600 \mathrm{MHz}\right.$; DMSO- $\left.d_{6}\right) 13.68(1 \mathrm{H}, \mathrm{s}, \mathrm{NH})$, $8.62(1 \mathrm{H}, \mathrm{d}, J 8.0 \mathrm{~Hz}, \mathrm{ArH}), 8.38(1 \mathrm{H}, \mathrm{d}, J 8.8 \mathrm{~Hz}, \mathrm{ArH}), 8.21(1 \mathrm{H}, \mathrm{d}, J 7.3 \mathrm{~Hz}, \mathrm{ArH}), 8.09(2 \mathrm{H}, \mathrm{d}, J 13.4 \mathrm{~Hz}, \mathrm{ArH})$, $7.98(1 \mathrm{H}, \mathrm{d}, J 8.8 \mathrm{~Hz}, \mathrm{ArH}), 7.80(1 \mathrm{H}, \mathrm{s}, \mathrm{ArH}), 7.7-7.6(5 \mathrm{H}, \mathrm{m}, \mathrm{ArH}), 7.55(1 \mathrm{H}, \mathrm{d}, J 8.6 \mathrm{~Hz}, \operatorname{ArH}), 7.28(2 \mathrm{H}, \mathrm{s}, \mathrm{ArH})$ and $6.77(1 \mathrm{H}, \mathrm{d}, J 13.4 \mathrm{~Hz}, \mathrm{ArH})$; $\delta \mathrm{c} / \mathrm{ppm}\left(150 \mathrm{MHz}\right.$; DMSO- $\left.d_{6}\right) 157.3$ and $155.8(\mathrm{C}=0), 148.9,148.0,145.8$, 144.7, 137.5, 136.5, 134.0, 132.1, 132.0, 131.32, 131.31, 130.8, 130.3, 129.5, 129.1, 128.9, 128.5, 128.4, 125.4, 124.9, 124.0, 121.8, 121.3, 115.3 and 112.8 (ArC). Note. Doubling of certain C-13 signals attributed to rotamerism of the amide moiety.

\section{Bioassay protocols}

Cytotoxicity determination. To assess the overt cytotoxicity of the compounds, HeLa (human cervix adenocarcinoma) cells cultured in DMEM medium containing $10 \%$ fetal bovine serum and antibiotics (penicillin, streptomycin, amphotericin $\mathrm{B}$ ) in a $5 \% \mathrm{CO}_{2} 37^{\circ} \mathrm{C}$ incubator were plated in 96 -well plates at a density of $2 \times 10^{4}$ cells per well. After an overnight incubation to allow cell adhesion, test compounds were added to a final concentration of $20 \mu \mathrm{M}$ and incubation continued for 48 hours. Residual cell viability was determined by adding resazurin to a final concentration of $50 \mu \mathrm{M}$ and measuring fluorescence (Ex $\mathrm{x}_{50} / \mathrm{Em}_{590}$ ) in a Spectramax M3 plate reader (Molecular Devices) after a 4-hour incubation. Fluorescence readings were converted to \% cell viability in compound-treated wells relative to untreated controls, after subtracting background readings obtained from wells without cells. Compounds were tested in duplicate wells. Emetine was used as a control drug standard.

Anti-trypanosomal assay. To assess trypanocidal activity, T.b. brucei (strain 427) trypomastigotes were seeded in 96-well plates at a density of $2.4 \times 10^{4}$ cells per well in medium consisting of IMDM containing $25 \mathrm{mM}$ 
HEPES, 10\% fetal bovine serum, $1 \mathrm{mM}$ hypoxanthine, antibiotics (penicillin, streptomycin) and HMI-9 supplement. After an overnight incubation at $37^{\circ} \mathrm{C}$ in a $5 \% \mathrm{CO}_{2}$ incubator, compounds were added to a final fixed concentration of $20 \mu \mathrm{M}$ or as 3-fold serial dilutions, and incubation continued for 24 hours. Resazurin was added to a final concentration of $50 \mu \mathrm{M}$ and, after an additional 24-hour incubation, fluorescence (Ex $560 /$ Em $_{590}$ ) was measured in Spectramax M3 plate reader. Fluorescence readings were converted to \% parasite viability in compound-treated wells relative to untreated controls, after subtracting background readings obtained from wells without cells. Compounds were tested in duplicate wells. Pentamidine was used a control drug standard

\section{Acknowledgements}

The authors are indebted to the Tertiary Education Trust fund (TETfund) for a bursary (to K.A. Oluwafemi), Adekunle Ajasin University, Akungba-Akoko, Nigeria for study leave (for K.A. Oluwafemi) and Rhodes University, Makhanda/Grahamstown South Africa. This research project was supported by the South African Medical Research Council (SAMRC) and by the Rhodes University Sandisa Imbewu programme.

\section{Supplementary Material}

The ${ }^{1} \mathrm{H}$ - and ${ }^{13} \mathrm{C}-\mathrm{NMR}$ spectra for all new compounds $\mathbf{8}, \mathbf{4 a -} \mathbf{g}$, and $\mathbf{5 h}, \mathbf{i}$ and the bioassay reports and data are provided in the Supplementary Material file.

\section{References}

1. Malvy, D. ; Chappuis F. Clin Microbiol Infect., 2011, 17, 986-995. https://doi.org/10.1111/j.1469-0691.2011.03536.x

2. Pollitt L.C.; MacGregor, P.; Matthews, K.; Reece, S.E. Trends Parasitol. , 2011, 27, 197-203. https://doi.org/10.1016/j.pt.2011.01.004

3. Simarro, P.P.; Jannin, J.; Cattand, P. PLOS medicine. 2008, 5, e55. https://doi.org/10.1371/journal.pmed.0050055

4. Wilkinson, S.R.; Kelly, J.M. Expert Reviews in Mol Med., 2009, 11, e31. https://doi.org/DOI org/10.1017/S1462399409001252

5. Barrett, MP PLoS pathog., 2010, 6, e1001204. https://doi.org/DOI org/10.1371/journal.ppat.1001204

6. Fairlamb. A.H. Trends Parasitol., 2003, 19, 488-494. https://doi.org/DOI 10.1016/j.pt.2003.09.002

7. Scotti, L.; Mendonça, F.J.B.; da Silva, M.S.; Scotti, M.T. Curr Protein Pept Sci., 2016, 17(3), 243-259 https://doi.org/10.2174/1389203717999160226173754

8. Baker, N.; de Koning, H.P.; Mäser, P.; Horn, D. Trends Parasitol., 2013, 29, 110118. https://doi.org/DOI 10.1016/i.pt.2012.12.005 
9. Chitanga, S.; Marcotty, T.; Namangala, B.; den Bossche P.V.; Abbeele, J.V.D.; Delespaux, V. PLoS Neg/ Tropl Dis., 2011, 5(12), e1454.

https://doi.org/10.1371/journal.pntd.0001454

10. Foley, M.; Tilley, L. Pharmacol Ther.,. 1998, 79, 55-87. https://doi.org/10.1016/s0163-7258(98)00012-6

11. O'Neill, P.M.; Bray, P.G.; Hawley, S.R.; Ward, S.A.; Park, B.K. Pharmacol Ther., 1998, 77, $29-58$. https://doi.org/10.1016/s0163-7258(97)00084-3

12. Homewood, C.A.; Warhurst, D.C.; Peters, W.; Baggaley, V.C. Nature 1972, 235, 50-52. https://doi.org/10.1038/235050a0

13. Hawley, S.R.; Bray, P.G.; Mungthin, M.; Atkinson, J.D.; O’Neill, P.M.; Ward, S.A. Antimicrob Agents Chemother., 1998, 42, 682-686. https://doi.org/10.1128/AAC.42.3.682

14. Roberts, L.; Egan, T.J.; Joiner, K.A.; Hoppe, H.C. Antimicrob Agents Chemother., 2008, 52, 1840-1842. https://doi.org/10.1128/AAC.01478-07

15. Ressurreição, A.S.; Gonçalves, D.; Sitoe, A.R.; Albuquerque, I.S,.; Gut, J.; Góis, A.; Gonçalves, L.M.; Bronze, M.R.; Hanscheid, T.; Biagini, G.A.; Rosenthal, P.J.; Prudêncio, M.; O'Neill, P.; Mota, M.M.; Lopes, F.; Moreira, R. J. Med. Chem., 2013, 56, 7679-7690. https://doi.org/10.1021/jm4011466

16. Rodrigues, T.; da Cruz, F.P.; Lafuente-Monasterio, M.J.; Gonçalves, D.; Ressurreição, A.S.; Sitoe, A.R.; Bronze, M.R.; Gut, J.; Schneider, G.; Mota, M.M.; Rosenthal, P.J.; Prudêncio, M.; Gamo. F; Lopes, F.; Moreira, R. J. Med Chem., 2013, 56, 4811-4815. https://doi.org/10.1021/jm400246e

17. Sankaran, M.; Chandraprakash, K.; Uvarani, C.; Vennila, K.; Velmurugan, D.; Mohan, P. Synlett. 2012, 23, 2858-2864.

https://doi.org/10.1055/s-0032-1317488 